\title{
Comparison of Shielding Effectiveness in Complex Curved Structure with Different Numerical Methods, FDTD, MOM and Equivalent Circuit
}

\author{
A. H. Poursoltan Mohammadi ${ }^{1}$, M. Chehel Amirani ${ }^{2}$ Faghihi $^{3}$ \\ ${ }^{1,2}$ Faculty of Electrical and Computer Engineering Urmia University, Urmia-Iran \\ ${ }^{3}$ Science and Research Branch, Islamic Azad University, Tehran-Iran
}

\begin{tabular}{l} 
Article Info \\
\hline Article history: \\
Received Jun 8, 2018 \\
Revised Aug 20, 2018 \\
Accepted Sep 1, 2018 \\
\hline
\end{tabular}

Keywords:

Apertures

Complex structure

Electromagnetic interference

Shielding effectiveness

\begin{abstract}
The study of the effect of shielding on high frequency equipment is very important in the electromagnetic compatibility of control and communication equipment. In this paper, while presenting a curved complex structure for the shielding enclosure, the different number of apertures with different dimensions has been investigated. A rectangular structure with two curved parts behind of the enclosure simulated based on numerical methods, FDTD, MOM and equivalent circuit for better analysis of electromagnetic interference. After introducing the proposed structure and presenting the curvature theory, simulation results are displayed and compared in the selected frequency range for three numerical methods. It has been shown that increasing the number of apertures by reducing the size, increases the effectiveness of the protective shield. However, increasing the number of resonances by increasing the apertures indicates the importance of studying the equipment more precisely before choosing the structure of enclosure. We present a complex structure for the enclosure and the different number and dimensions of apertures with different materials were investigated for analyzing the effect of shielding on electromagnetic interference. The necessity of choosing a more effective enclosure according to the frequency of the equipment is specified. Finally, three methods of numerical solution, FDTD, MOM and circuit equal comparition were performed with measured value. Changes in the Shielding effectiveness and the number of resonant in the frequency range were determined. The exact examination of equipment requires shielding and their frequency and the type of inside-to-outside communication device before choosing shieldin is important. We used a comparison of three numerical solution methods for examining the field distribution in a complex structure enclosure with different apertures and different materials. In the majority of cases, the proximity of the measured values in this frequency range with the MOM curves shows the performance of this method in complex structures.
\end{abstract}

Copyright $@ 2018$ Institute of Advanced Engineering and Science. All rights reserved.

\section{Corresponding Author:}

A. H. Poursoltan mohammadi,

Faculty of Electrical and Computer Engineering,

Urmia University, Urmia-Iran

Email: ah.poursoltan@gmail.com

\section{INTRODUCTION}

At present, the use of metal enclosures has increased for devices operating at high frequency [1]. In the frequency of operation, if the wavelength range and the dimensions of the enclosure are close, the effect of the enclosure in the interference should be well investigated. Electromagnetic interference (EI) is a complex, continuous and random signal, and requires accurate analysis and measurement [2]. In fact, 
the presence of apertures in a metal enclosure is due to the presence of connectors and communication cables in the shielding enclosure. Different methods of numerical solution are used to compute Shielding effectiveness (SE) in complex compartments [3]. To calculate the SE in electromagnetism, numerical solution methods such as Method of Moments (MOM), Finite-difference time-domain (FDTD) method and Equivalent Circuit Methods (ECM) are used. Solving the integral equations in electromagnetic with using numerical methods is studied for more than 4 decades. Simple shapes in the form of square and rectangular cylinders, circles, etc. have been analyzed over decades [4]. The use of basic functions in many of these numerical methods and transforming the integral equation into a linear system and finally, solving it with direct methods and repetition is the ideal solution to achieve the desired goal [5]. The simplest method for solving electromagnetic problems is the FDM numerical solution method, which converts the differential equation into partial differential equations. In this way, the whole area is divided into nodes. The differential approximation of the original equation is written for them and solved with using algebraic algebraic equations matrix. This matrix is very large after the solution and does not support the open region, which is one of the important problems of this solution [6-7]. FDTD is a hybrid numerical method and used to solve electromagnetic problems. Because the rigorous numerical method and the asymptotic scheme do not work well, the combined method is more effective in solving problems in complex structures [8-10]. In the method of moments, as an advanced numerical solution, instead of using Maxwell differential equations, the Integral responses of Maxwell's Equations are used. For this reason, If Green's function is known, the function of the MOM method is excellent for solving electromagnetic problems in complex structures [4] and [11]. To analyze the field distribution on the outer and inner surfaces of a metal enclosure, method of moment numerical method is an efficient method. In this method, by applying boundary conditions, the accuracy of field calculations has been improved [12]. An electromagnetic compatibility analysis of the components inside the enclosure is easy for simple structures with ordinary geometric shapes. However, the precise analysis of the performance of components in the complex structure with apertures is difficult and complex in terms of electromagnetic interference. So it needs to be reviewed and computed. With the advancement of technology, the growth of electronic equipment is faster than before [13]. Electromagnetic interference has become more and more complex in the environments, and as a result, the importance of electromagnetic compatibility analysis has increased.

For this reason, the performance analysis of complex structures with different number of apertures and different shapes will provide a more accurate understanding of the destructive effects of electromagnetic interference. The enclosure with complex structure, with different dimensions of apertures, has been studied and compared with different numerical methods to investigate the interference effects of the equipment installed in the enclosure. The investigated shielding can create good conditions for accurate operation of high-frequency circuits in compressed and confined spaces $[1,2]$.

\section{ANALYZING SHIELDING EFFECTIVENESS WITH DIFFERENT NUMERICAL METHODS}

At present, different shapes of metal enclosures are used to reduce electromagnetic interference. Shielding effectiveness is an important parameter for design and selection of the enclosure. This coefficient can be calculated by using equations 1 and 2 [12], [14].

$$
\begin{aligned}
& S E_{E}=20 \log _{10}\left|\frac{E_{i}}{E_{t}}\right| d B \\
& S E_{B}=20 \log _{10}\left|\frac{H_{i}}{H_{t}}\right|
\end{aligned}
$$

\subsection{Investigation of Electromagnetic Interference in Complex Curved Structure with Apertures by Equivalent Circuit Method}

The complex structure of the enclosure is shown in Figure 1. Considering the appearance of these enclosures, it can be expected that conditions will be provided to use functional and compact enclosures instead of simple rectangular and square enclosures in the future. The internal curvature in both sides of the enclosures and the different number of apertures makes the analytical calculations complex and specific. The apertures act as the actual model for the arrival and departure of communication equipment in the enclosures. The effect of this structural form on the electric field and the intensity of the field are shown in Figure 2 [15]. The specification of the enclosure wall includes is, $\mathrm{K}$ the conductivity, $\delta$ effect of the wall thickness, and $\varepsilon_{\mathrm{r}}$ the relative permeability coefficient. For exciting two short monopoles Antenna are used in the coordinates $\left(\mathrm{x}_{1}, \mathrm{y}_{1}, 0\right)$ and $\left(\mathrm{x}_{2}, \mathrm{y}_{2}, 0\right)$. $\mathrm{L}$ is length of the Antenna and $\mathrm{r}$ is the radius of the Antenna. The characteristics of the probes and Parameters for design of the enclosure are shown in Table 1. We use Coaxial cable for supply [16]. Metal shielding is one of the main tools for preventing electromagnetic

Comparison of Shielding Effectiveness in Complex Curved Structure with... (A. H. Poursoltan mohammadi) 
interference, which is a guarantee of electromagnetic compatibility in electronic equipment. SE coefficient specifies the shielding efficiency of a metal enclosure. The presence of apertures on the wall of enclosure changes the behavior of the shields.

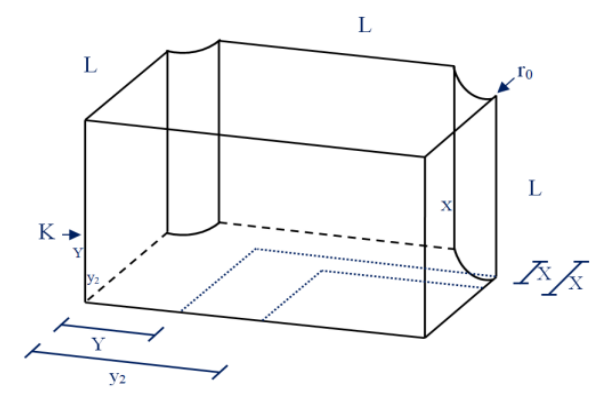

Figure 1. The Complex Curved Structure with Apertures

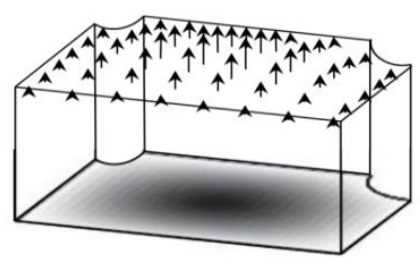

(a)

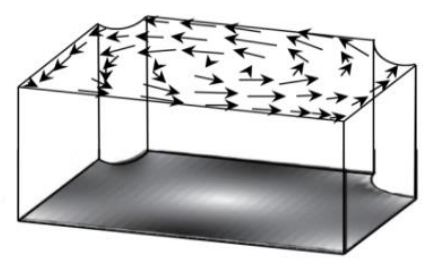

(b)

Figure 2. Distribution of magnetic (a) and electric (b) field in a complex special structure

Table 1. Characteristics complex structure enclosure

\begin{tabular}{ccc}
\hline Caviti & Current Probe 1 & Current Probe 2 \\
\hline $\mathrm{L}_{\mathrm{x}}=400 \mathrm{~mm}$ & $\mathrm{X}_{1}=90 \mathrm{~mm}$ & $\mathrm{X}_{2}=120 \mathrm{~mm}$ \\
$\mathrm{~L}_{\mathrm{y}}=500 \mathrm{~mm}$ & $\mathrm{y}_{1}=110 \mathrm{~mm}$ & $\mathrm{y}_{2}=300 \mathrm{~mm}$ \\
$\mathrm{~L}_{\mathrm{z}}=300 \mathrm{~mm}$ & $\mathrm{~L}_{1}=1 \mathrm{~mm}$ & $\mathrm{~L}_{2}=1 \mathrm{~mm}$ \\
$\mathrm{r}_{0}=100 \mathrm{~mm}$ & & \\
$\epsilon_{\mathrm{r}}=2.2$ & & \\
$\mathrm{k}=10^{5} \mathrm{~s} / \mathrm{m}$ & & \\
\hline
\end{tabular}

The simulation of the SE coefficient in complex structures with apertures is complicated by considering the solution of waveguide. Therefore, it is necessary to use the best method for calculating the SE coefficient in enclosure when resonance problems occur for large electric fields [3]. The evaluation and computation of field parameters at common boundaries between two different physical environments is necessary. The boundary conditions are shown in Figure 3.

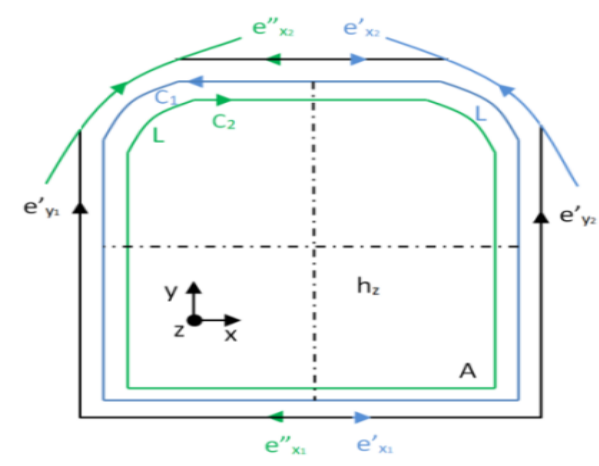

Figure 3. Boundary conditions in a complex structure 
The tangential field components $\mathrm{E}$ are continuous along the whole boundary (Equation 3). The Vertical field components is calculated at the boundary of the two materials of Equation 9, where D is the density of the flux and $\rho$ is the surface electrical charge density $[18,19]$.

$$
\begin{aligned}
& \begin{array}{l}
\nabla . D=\rho \\
\nabla \times E=0 \\
E_{1 t}=E_{2 t} \quad\left(\frac{V}{m}\right) \\
D_{1 n}-D_{2 n}=\rho_{s} \quad\left(\frac{C}{m^{2}}\right) \\
e_{i}^{\prime}=e^{\prime \prime}=\int_{L_{i}} E . d s, \quad d_{i}=\int_{\bar{A}_{i}} D . d A, \quad q_{i}=\int_{\bar{V}_{i}} \rho d V
\end{array}
\end{aligned}
$$

The boundary conditions of $\mathrm{B}$ and $\mathrm{H}$ vectors analysis are necessary in environments with different physical properties. The static magnet is characterized by fundamental equations as Equations 8 . Since divergence of B is zero in Equation 9, we conclude that the vertical component of B is continuous to crosses the boundaries. By the integral form of the Kearl equation, the boundary conditions of the tangential magnetic field component are calculated from Equation 10, which $\mathrm{J}_{\mathrm{sn}}$ is the surface flow density [18-20].

$$
\begin{aligned}
& \nabla \times H=0 \\
& B_{1 n}=B_{2 n} \\
& H_{1 t}-H_{2 t}=J_{s n} \quad\left(\frac{A}{m}\right) \\
& h^{\prime}{ }_{i}=h^{\prime \prime}{ }_{i}=\int_{L_{i}} H . d s, \quad d_{i}=\int_{\bar{A}_{i}} B . d A, \quad j_{i}=\int_{\bar{A}_{i}} J d A \\
& C h=\frac{d}{d t} d+j, \quad C e=-\frac{d}{d t} b, \quad S b=0, \quad S d=q
\end{aligned}
$$

In the equivalent circuit method, the curved volume at the behind of the complex structure is excited by current probes. The equivalent impedance between these probes is obtained by using the power balance. Analysis of the Helmholtz equation for a curved surface with a volume $\mathrm{V}$ and a contact surface $\mathrm{S}$ has been performed. The electric Field is divided in two sections, a rotational part with a zero divergence $\left(E_{n}\right)$ and a non-rotational Section with a zero Curl $\left(\mathrm{F}_{\mathrm{m}}\right)$. Finally, the total magnetic field is computed and displayed. The total electric field equation is given (Equation 13). Since the non-rotational part is zero, we need to study the rotational section. This formula based on the Helmholtz equation is as follows (Equation 14). Given Equations (13) and (14), Equation (15) is obtained. Because $E_{n}$ and $F_{m}$ are orthogonal, equations 16 and 17 are calculated. Using the electric field expansion model (Equation 13) and using Equations 16 and 17, we can calculate the total impedance of the whole system $\left(\mathrm{Z}_{\mathrm{IJ}}\right)$ as a two-port network with ports I and J, which is shown in Equation 19.

$$
\begin{aligned}
& E=\sum_{0}^{\infty} e_{n} E_{n}+f_{m} F_{m} \\
& \nabla \times \nabla \times E-K^{2} E=-j \omega \mu J \\
& \sum_{n=0}^{\infty}-\frac{j \omega \mu}{\left(k_{n}^{2}-k^{2}\right) K_{E, n}}<J, E_{n}>\left(k_{n} E_{n}-k^{2} E_{n}\right)=-J \omega \mu J \\
& e_{n}=\frac{j \omega \mu}{\left(k_{n}^{2}-k^{2}\right) K_{E, n}}<J, E_{n}> \\
& f_{n}=\frac{1}{j \omega \varepsilon K_{F, n}}<J, F_{n}> \\
& Z_{i j}=\frac{j \omega \mu}{I_{j} I_{i}^{*}} \sum_{n=0}^{\infty} \frac{<E_{n}, J_{j}><E_{n}, J_{i}^{*}>}{\left(k_{n}^{2}-k^{2}\right) K_{E, n}}+\frac{1}{j \omega \varepsilon I_{j} I_{i}^{*}} \sum_{n=0}^{\infty} \frac{<F_{n}, J_{j}><F_{n}, J_{i}^{*}>}{K_{F, n}}
\end{aligned}
$$




\subsection{Shielding effectiveness by FDTD Method}

Finite Difference Time Domain Method (FDTD) is an efficient method for solving Maxwell's equations. The main weakness of this method is the divergence error, which affects the accuracy of the numerical solution. The presence of stairs and curves in complex structures has a negative effect on the convergence of this method. To solve this problem, the combination of this method with the HO method (higher order FDTD) is used [15]. In Figure 4. the coordinates of the enclosure in the boundary region are visible.

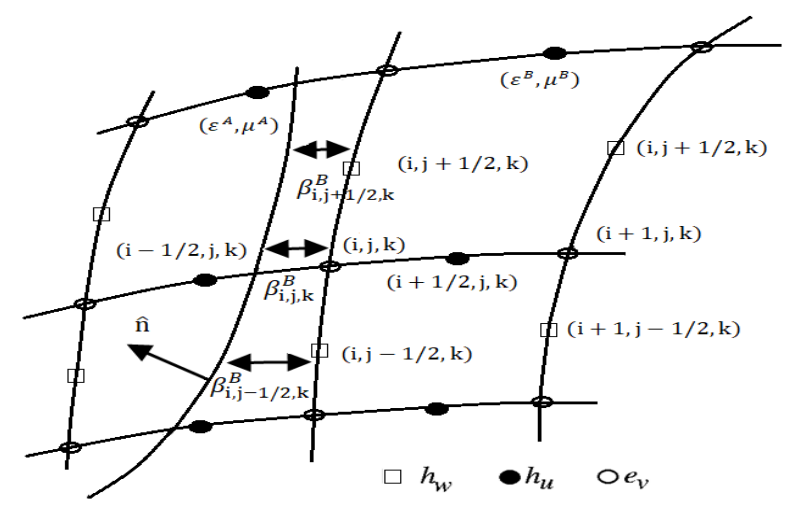

Figure 4. Coordinates of the enclosure in the Boundary region

It is assumed that the boundary specified with $\hat{n}=\left(\hat{n}_{u}, \hat{n}_{v}, \hat{n}_{w}\right)$ in Figure 4 is a normal unit vector. The components of the covariance of the electric and magnetic fields $\mathrm{E}_{\mathrm{cv}}^{\mathrm{mt}}$ and $\mathrm{H}_{\mathrm{cv}}^{\mathrm{mt}}$ on the boundary between the two regions $\mathrm{A}$ and $\mathrm{B}$ and for the values of $\mu^{\mathrm{mt}}$ and $\varepsilon^{\mathrm{mt}}$ are calculated as follows.

$$
\begin{array}{ll}
\hat{n} \times E_{c v}^{A}=\hat{n} \times E_{c v}^{B} & \varepsilon^{A} \hat{n} \cdot E_{c v}^{A}=\varepsilon^{B} \hat{n} \cdot E_{c v}^{B} \\
\hat{n} \times H_{c v}^{A}=\hat{n} \times H_{c v}^{B} & \varepsilon^{A} \hat{n} \cdot H_{c v}^{A}=\varepsilon^{B} \hat{n} \cdot H_{c v}^{B}
\end{array}
$$

If in the FDTD method, we assign the $(\mathrm{i}, \mathrm{j}, \mathrm{k}) \equiv(\mathrm{i} \Delta \mathrm{u}, \mathrm{j} \Delta \mathrm{v}, \mathrm{k} \Delta \mathrm{w})$ character to the cell's coordinates as in Figure 4. We assign a parameter to each cell with respect to its distance from the wall. This coefficient $\beta_{\mathrm{i}, \mathrm{j}, \mathrm{k}}^{\mathrm{mt}}$ depends on the geometric shape of the boundaries. By applying the central finite difference and the above definitions, the discrete form of the Maxwell equations is as follows. These formulas are presented for $\mathrm{H}_{\mathrm{u}}$ in two regions $\mathrm{A}$ and $\mathrm{B}$. We extend $\mathrm{H}_{\mathrm{u}}^{\mathrm{B}}$ by using Equation 21. All Maxwell equations are solved by numerical methods, and the analysis of electromagnetic interference by the SE is possible [15].

$$
\begin{aligned}
& H_{u}^{A}(i, j, k)=\left(1+\beta_{i, j, k}^{A}\right) H_{u}\left(i-\frac{1}{2}, j, k\right)+\beta_{i, j, k}^{A} H_{u}\left(i-\frac{3}{2}, j, k\right) \\
& 4 H_{u}^{A}(i, j, k)=\breve{H}_{u}\left(i, j-\frac{1}{2}, k-\frac{1}{2}\right)+\breve{H}_{u}\left(i, j+\frac{1}{2}, k-\frac{1}{2}\right)+\breve{H}_{u}\left(i, j+\frac{1}{2}, k+\frac{1}{2}\right)+\breve{H}_{u}\left(i, j-\frac{1}{2}, k+\frac{1}{2}\right) \\
& 2 H_{u}^{B}(i, j, k)=\breve{H}_{u}\left(i, j+\frac{1}{2}, k\right)+\breve{H}_{u}\left(i, j-\frac{1}{2}, k\right) \\
& H_{u}^{B}(i, j, k)=\breve{H}_{u}\left(i, j+\frac{1}{2}, k\right)+\frac{\Delta t}{\mu^{B}}\left(\frac{E_{v}\left(i, j+\frac{1}{2}, k+1\right)-E_{v}\left(i, j+\frac{1}{2}, k\right)}{\Delta w}-\frac{E_{w}\left(i, j+\frac{1}{2}, k+1\right)-E_{v}\left(i, j, k+\frac{1}{2}\right)}{\Delta v}\right)
\end{aligned}
$$

\subsection{Shielding Effectiveness by MOM Method:}

The curved section in this enclosure needs accurate analysis and simple numerical methods will not be responsive. The MOM numerical solution method can solve this problem. The way of solving in this method is the basic equations of electromagnetic fields, which fields are obtained in a boundary condition by numerical solution of these equations. Finally, solving the matrix (depending on the type of problem and its convergence) is done by numerical solution. To solve the divergence problem of the integral expansion at high frequencies, the combination of physical optics and MOM is used in simulation. This simulation has been performed at 0-3 gigahertz of frequency range so we can say that the combination of MOM and 
physical optics will respond to the simulation of electromagnetic fields in complex structure at high frequencies [17]. The field equation can be written as Equation 25 whit considering the existence of $E_{i}^{i}\left(-M_{s}\right)+E_{t}^{i}\left(J^{i}\right)=0$ in the enclosure and $E_{t}^{0}\left(J^{i n c}\right)+E_{t}^{0}\left(M_{s}\right)+E_{t}^{0}\left(J^{0}\right)=0$ out of the enclosure. Helmholtz's equation for correction of electric field is used in two parts of rotational with a zero divergence $\left(E_{n}\right)$ and non- rotational with a zero Curl $\left(F_{m}\right)$. The magnetic field and total electric field are calculated and represented with using the Helmholtz equation. The non-circular part is empty, so the study of the circulation section is sufficient. The basic idea of MOM is that the unknown quantity $\mathrm{f}$ is expanded to a set of Linear Independent Functions which is approximated by the following limited series (Equation 26). $\alpha_{\mathrm{n}}$ is the weight factor. After considering the boundary conditions, we define the internal multiplication or a moment between the fundamental function $\mathrm{f}_{\mathrm{n}}\left(\mathrm{r}^{\prime}\right)$ and the weight function $\mathrm{f}_{\mathrm{m}}(\mathrm{r})$ which is shown in Equation 27 [12, 16].

$$
\begin{aligned}
& H_{t}^{0}\left(J^{i n c}\right)+H_{t}^{0}\left(M_{s}\right)+H_{t}^{0}\left(J^{0}\right)=H_{i}^{i}\left(-M_{s}\right)+H_{t}^{i}\left(J^{i}\right) \\
& f \approx \sum_{n=1}^{N} \alpha_{n} f_{n} \\
& \left\langle f_{m}, f_{n}\right\rangle=\int_{f_{m}} f_{m}(r) \cdot \int_{f_{n}} f_{n}\left(r^{\prime}\right) d r^{\prime} d r
\end{aligned}
$$

\section{SIMULATION AND COMPUTING}

Various magnetic materials such as iron, copper and zinc are commonly used for equipment as passive shielding. The accurate use of the shielding method can be used as a way to reduce and eliminate the destructive effects of electromagnetic fields on equipment. Shielding can be placed around circuits, systems, wires or communication cables [23]. The reduction of the radiant electromagnetic field in the shielding is determined by the SE coefficient [24-27]. An enclosure with dimensions of $20 * 20 * 40$ is simulated with the aperture and the simulation of Shielding effectiveness is performed by using three methods of numerical solution, FDTD, MOM and equivalent circuit in a Curved enclosure with Apertures. This simulation is performed in four steps in the enclosure. One time, this simulation was performed with a large $10 * 20 \mathrm{~cm}$ wide aperture (Figure 5) and then with 12, 36 and 60 small apertures (Figure 7-9). The SE curve for the magnetic and electric field is shown in Figure 6. Finally, the values of different numerical solving methods are compared with the measured values and are shown in Figure 10.

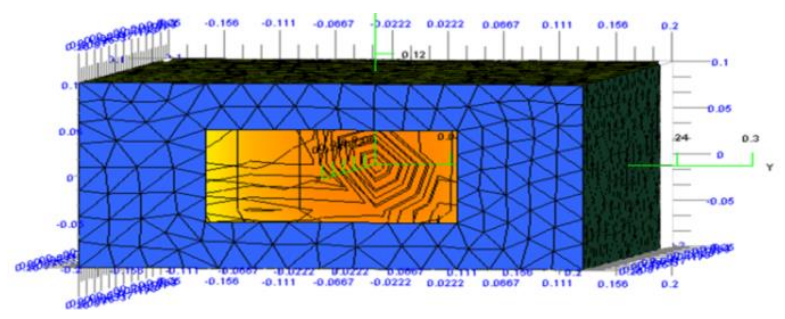

Figure 5. Rectangular enclosure with a large aperture in its center
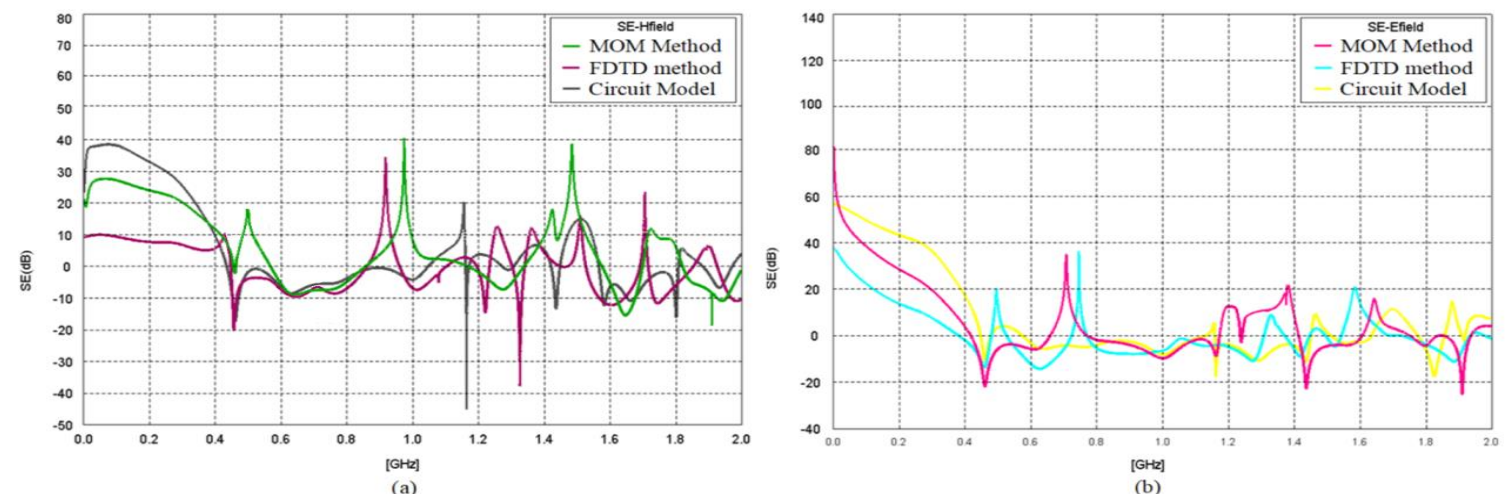

Figure 6. SE curve for (a) the magnetic field (b) the electric field 


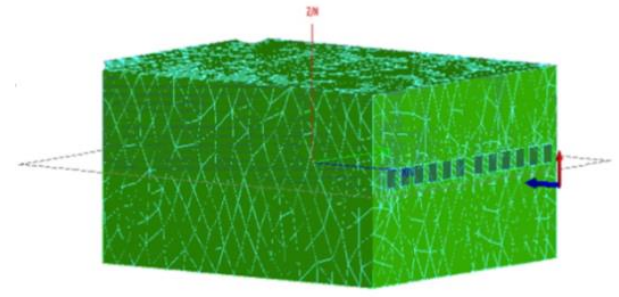

(a)

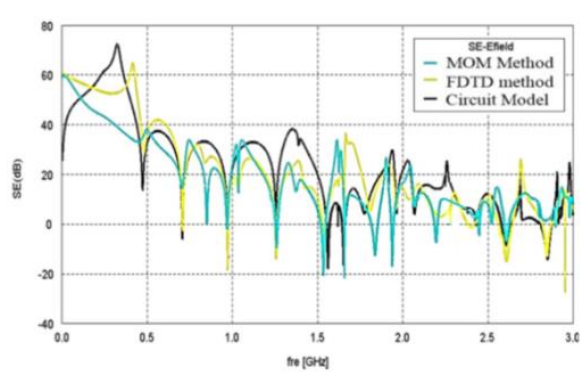

(b)

Figure 7. Enclosure with 12 apertures $(2 * 2 \mathrm{~cm})(\mathrm{b}) \mathrm{SE}$ curve

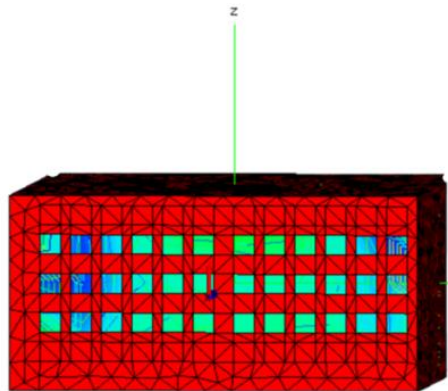

(a)

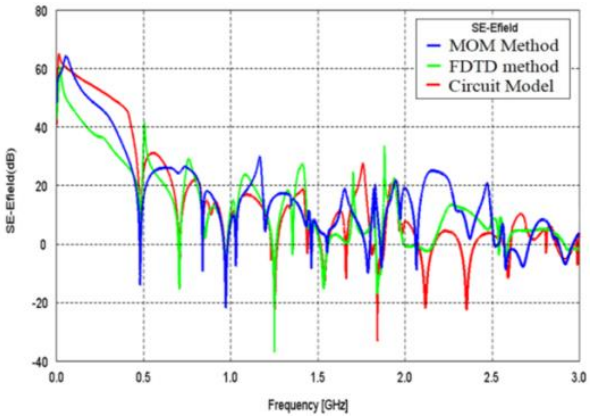

(b)

Figure 8. Enclosure with 36 apertures $(2 * 2 \mathrm{~cm})$ (b) SE curve
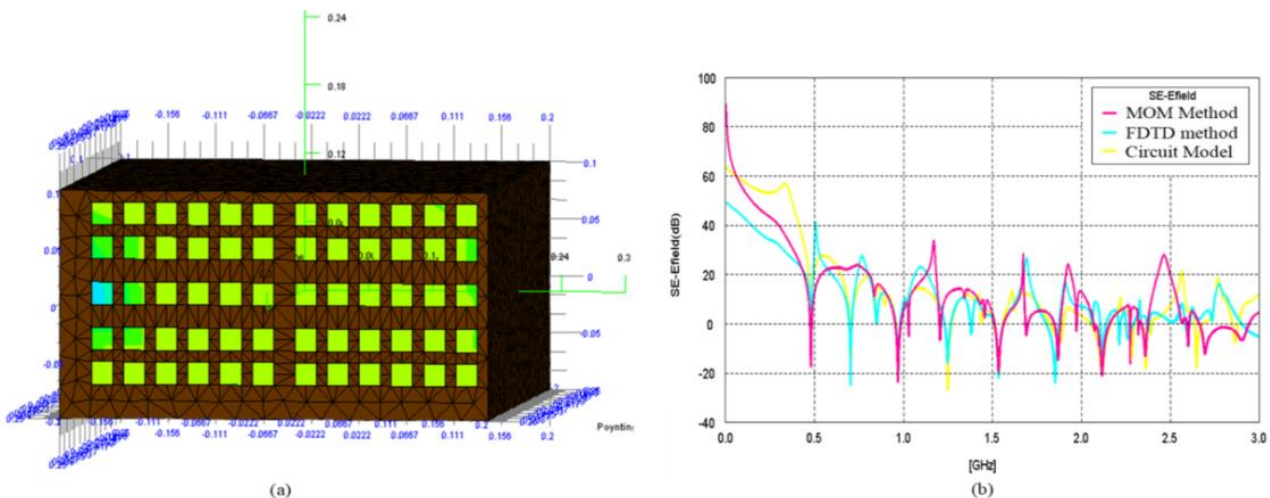

Figure 9. Enclosure with 60 apertures $(2 * 2 \mathrm{~cm})(\mathrm{b}) \mathrm{SE}$ curve

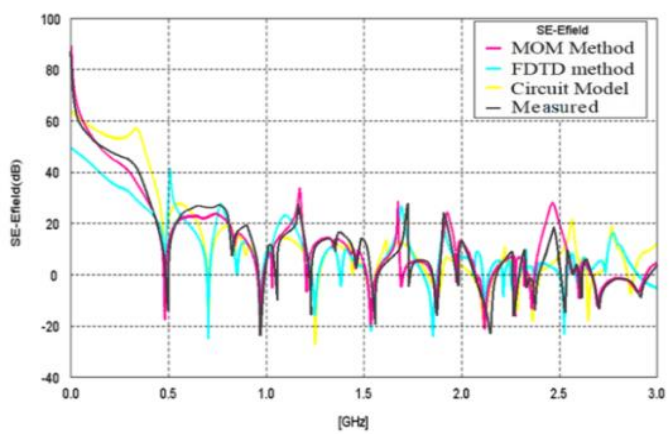

Figure 10. Comparison between three numerical solution methods with measured values of SE curve with 60 apertures $(2 * 2 \mathrm{~cm})$ 
In the final section, we attempt to evaluate the SE with using different materials and measure their impact on the quality of the enclosure efficiency. We have chosen three materials, Iron, Copper and Mu-Metal. Simulation of SE curves with different materials is shown in Figures 11-13.
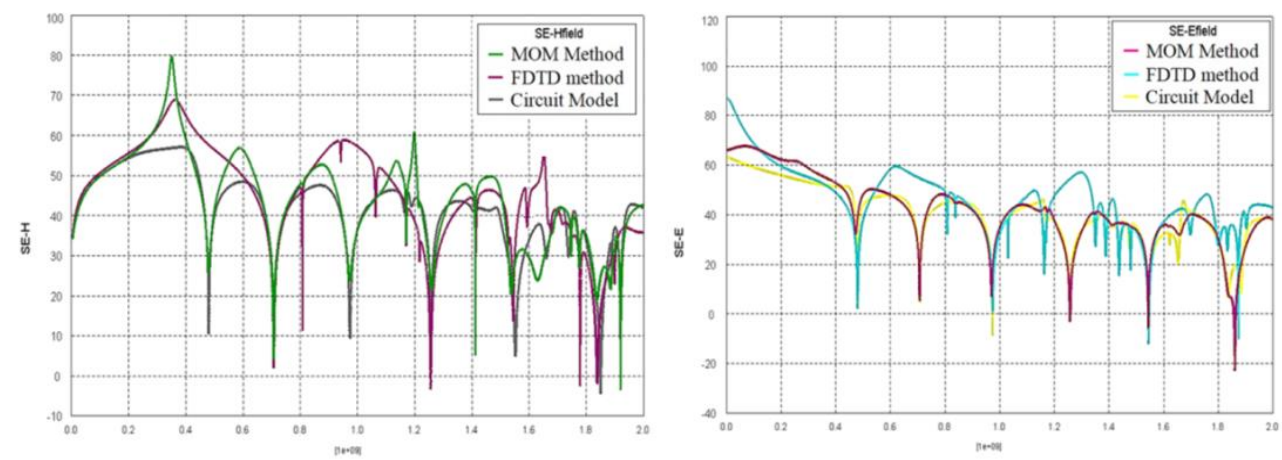

Figure 11. SE E and H field curves in the Iron enclosure
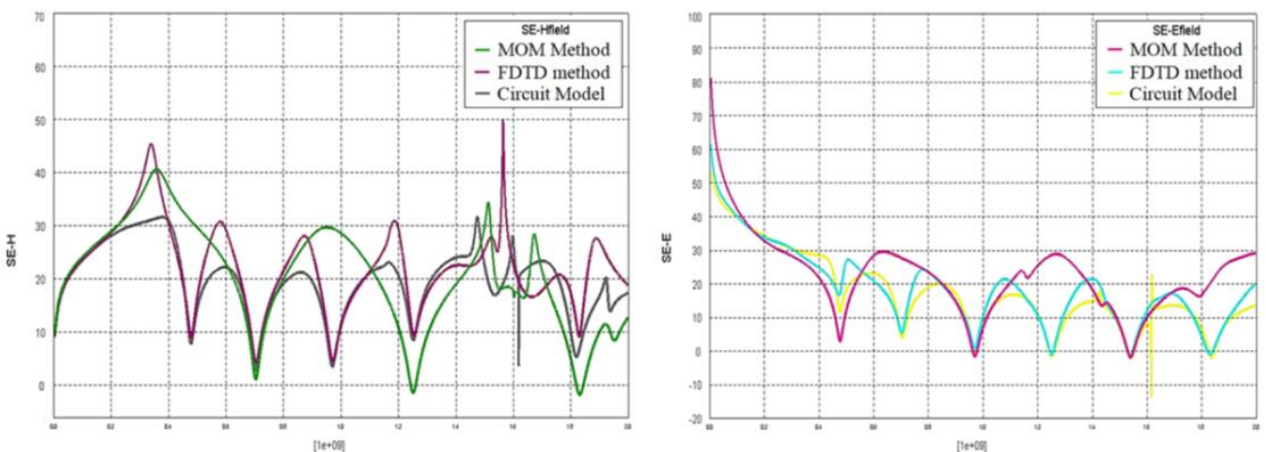

Figure 12. SE E and $\mathrm{H}$ field curves in the Mu-Metal enclosure
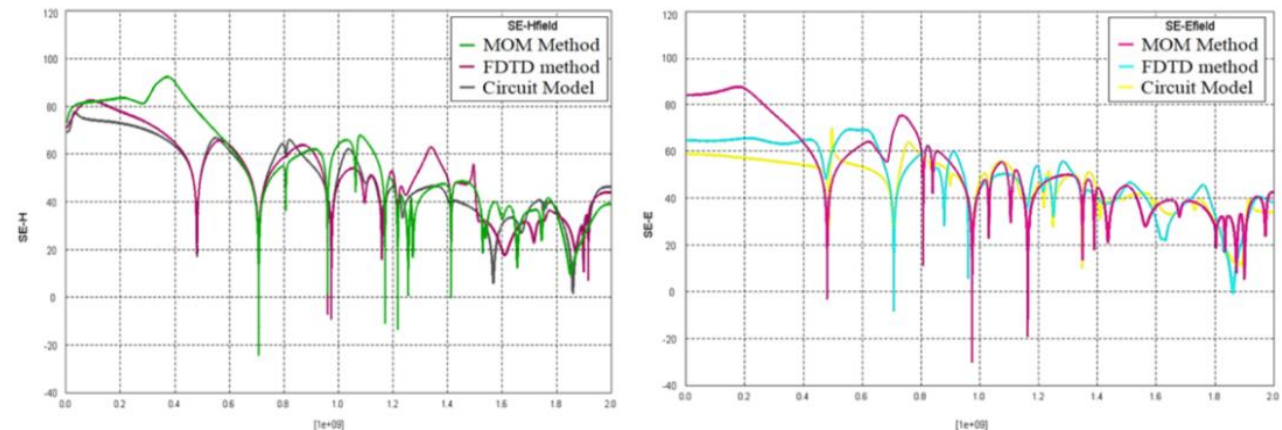

Figure 13. SE E and $\mathrm{H}$ field curves in the Copper enclosure

\section{CONCLUSION}

In this paper, the effect of different apertures and material on the shielding effectiveness in a complex structure has been accomplished by three methods of numerical solution, FDTD, MOM and equivalent circuit. The complex structure of the enclosure, despite its curvature in the enclosure, allows the equipment to be minimized and optimizes the dimensions. in fact, The use of apertures in the wall of the enclosure is due to the modeling of the connection of cables and ventilation. The enclosure simulation was performed to determine the shielding effectiveness in four different compartments and three different materials in frequency range of 0-3 gigahertz. What is clearly visible is the high level of effectiveness at low

Comparison of Shielding Effectiveness in Complex Curved Structure with... (A. H. Poursoltan mohammadi) 
frequencies in all three numerical methods. Increasing the number of apertures and decreasing its dimensions, makes the shielding better, but the number of resonance points also increases. The simulation shows that the MOM method is closer to the measured values for most of the selected frequency range, which explains on the accuracy of this method in complex structures.

The use of different materials in the production of the enclosure shows that the Mu-metal enclosure is better and the SE in this enclosure is optimally increased throughout the entire frequency range. The presence of resonant points in frequency range, The importance of choosing the exact frequency of equipment is shown.

\section{REFERENCES}

[1] Loya S, Khan H. Analysis Of Shielding Effectiveness In The Electric Field And Magnetic Field And Plane Wave For Infinite Sheet Metals. International Journal of Electromagnetics and Applications. 2007; 6(2): 31-41.

[2] Li W, Wei G, Pan X, Lu X. Electromagnetic Compatibility Prediction Method Under the Multifrequency in-Band Interference Environment. IEEE Transactions on Electromagnetic Compatibility. 2018; 6(2): 341-355.

[3] Dehkhoda P, Tavakoli A, Moini R. Fast Calculation of The Shielding Effectiveness For A Rectangular Enclosure Of Finite Wall Thickness And With Numerous Small Apertures. Progress In Electromagnetics Research. 2008; PIER 86: 241-250.

[4] Varmazyar S, Moghadas H M N, Masouri Z. A moment method simulation of Electromagnetic scattering from Conducting bodies. Progress in Electromagnetics Research. 2008; PIER 89: 99-119.

[5] Delves L M, Mohamed J L. Computational Methods for Integral Equations. Cambridge University Press. 1985; Cambridge.

[6] Toflove A, Hagness S C. Computation Electrodynamics: The Finite Difference Time Domain Method. Artech House, 2000; Norwood.

[7] Toflove A, Brodwin M E. Numerial Solution of Steady-State Electromagnetic Scattering Problems Using the TimeDependent Maxwell's Equations. IEEE Transactions on Microwave Theory Tech. 1975; 3(3): 623-730.

[8] Toflove A, Hagness S C. Time domain physical optics for the higher-Order fdtd modeling in electromagnetic Scattering from 3-d complex and combined multiple materials objects. Progress In Electromagnetics Research. 2009; PIER 95: 57-62.

[9] Wang M Y, Xu J, Wu J, Yan Y B, Li H L. FDTD study on scattering of metallic column covered by doublenegative metamaterial. Journal of Electromagnetic Waves. 2007; 21(14): 1905-1914.

[10] Faghihi F, Heydari H. A Combination of Time Domain Finite Element-Boundary Integral with Time Domain Physical Optics for Calculation of Electromagnetic Scattering Of 3-D Structures. Progress in Electromagnetics Research. 2008; PIER 79: 463-474.

[11] ebadi S, forooraghi k. Green's Function Derivation of an annular waveguide for Application in Method of Moment Analysis of Annular Waveguide Slot Antennas. Progress in electromagnetics research. 2009; PIER 89: 101-119.

[12] Poursoltanmohammadi A H, chehel Amirani M, Faghihi F. Presentation of an algorithm for secure data transmission based on optimal route selection during electromagnetic interference occurrence. International Journal of Electrical and Computer Engineering (IJECE). 2018; 8(1): 259-270.

[13] Faghihi F, Heydari H, Falahati A, Attar Y. Easy. Convolutional codes acting as emi virtual Shields in current injection systems. Progress in Electromagnetics Research. 2008; PIER 88: 337-353.

[14] Li G, Zhao X, Wen D, Yu Y. Research on Electromagnetic Excitation Resonant Sensor Based on Microelectromechanical System. TELKOMNIKA. Telecommunication. Computing Electronics and Control. 2015; 13(4), 71-79.

[15] Faghihi F, Heydari H. Time Domain Physical Optics For The Higher-Order Fdtd Modeling In Electromagnetic Scattering From 3-D Complex And Combined Multiple Materials Objects. Progress in Electromagnetics Research. 2009; PIER 95: 87-353.

[16] Lange C, Leone M. Broadband Circuit Model for Electromagnetic-Interference Analysisin Metallic Enclosures. IEEE Transactions on Electromagnetic Compatibility. 2018; 60(2), 368-375.

[17] Liu Q F, Yin W Y, Mao J F, Chen Z. Accurate characterization of shielding effectiveness of metallic enclosures with thin wires and thin slots. IEEE Transactions on Electromagnetic Compatibility. 2009; 51(2), 293-300.

[18] Makinen R M, Gersem H D. T Weiland. M Kivikoski. Modeling of Lossy Curved Surfaces in 3-D FIT/FDTD Techniques. IEEE Transactions on Antennas and Propagation. 2006; 54(11): 3490-3497.

[19] Dawson J F, Cabello M R, Alvarez J. Face Centered Anisotropic Surface Impedance Boundary Conditions in FDTD: Improved performance of staircased mesh for shielding problems. IEEE MTT-S International Conference on Numerical Electromagnetic and Multiphysics Modeling and Optimization for RF, Microwave, and Terahertz Applications, 2017.

[20] Graglia R D, ombard G L. Singular Higher Order Divergence-Conforming Bases of Additive Kind and Moments Method Applications to 3D Sharp-Wedge Structures. IEEE Transactions on Antennas and Propagation. 2008; 56(12): 3768-3788.

[21] Chen K, Ren D. An Improved MOM Approach to Determine the Shielding Properties of a Rectangular Enclosure with a Doubly Periodic Array of Apertures. IEEE Transactions on Electromagnetic Compatibility. 2016; 58(5): 1456-1464. 
[22] Boudkhil A, Chetioui M, Benahmed N. Development and Performance Enhancement of MEMS Helix Antenna for $\mathrm{THz}$ Applications using 3D HFSS-based Efficient Electromagnetic Optimization. 2018; TELKOMNIKA. Telecommunication. Computing. Electronics and Control. 16(1), 111-119.

[23] Faghihi F, Heydari H. Reduction of Leakage Magnetic Field In Electromagnetic Systems Based On Active Shielding Concept Verified By Eigenvalue Analysis. Progress in Electromagnetics Research. 2009; PIER 96: 217236.

[24] Azpurua M A, Silva A F. Decomposition of Electromagnetic Interferences in the Time-Domain. IEEE Transactions on Electromagnetic Compatibility. 2016; 58(2): 385-392.

[25] Beek S V, Leferink F. Vulnerability of Remote Keyless-Entry Systems against Pulsed Electromagnetic Interference and Possible Improvements. IEEE Transactions on Electromagnetic Compatibility. 2016; 58(2): 1259-1265.

[26] Loya S, Habibullakhan A. Analysis of Shielding Effectiveness in the Electric Field and Magnetic Field and Plane Wave for Infinite Sheet Metals. International Journal of Electromagnetics and Applications. 2016; 8(5): 40-46.

[27] Ghaloua A, Zbitou J, Zbitou J, Abdellaoui L, Latrach M, Errkik A. Reduction of Mutual Coupling between Closely Spaced Microstrip Antennas Arrays Using Electromagnetic Band-gap (2D-EBG) Structures. TELKOMNIKA, Telecommunication, Computing, Electronics and Control. 2018; 16(1): 31-41. 